\title{
Commentary on "Single random measurement of urinary gonadotropin concentration for screening and monitoring girls with central precocious puberty"
}

\author{
Ahreum Kwon \\ Department of Pediatrics, Severance \\ Children's Hospital, Yonsei University \\ College of Medicine, Seoul, Korea
}

See the article "Single random measurement of urinary gonadotropin concentration for screening and monitoring girls with central precocious puberty" via https://doi. org/10.6065/apem.2040208.104.

Address for correspondence: Ahreum Kwon

Department of Pediatrics, Severance Children's Hospital, Yonsei University College of Medicine, 50-1 Yonsei-ro, Seodaemun-gu, Seoul 03722, Korea

Email:armea@yuhs.ac https://orcid.org/0000-0002-96922135
This article ${ }^{1)}$ evaluates whether a single random measurement of urinary luteinizing hormone (LH) could diagnose the central precocious puberty (CPP) and monitor the effectiveness of the gonadotropin-releasing hormone (GnRH) agonist treatment. Because $\mathrm{CPP}$ is caused by premature activation of the hypothalamic-pituitary-gonadal axis, GnRH stimulation test is considered the gold standard for CPP diagnosis. However, the GnRH stimulation test is invasive, expensive, and relatively takes a long time of more than 2 hours. Therefore, many other simple tests including basal serum LH level, LH/follicle-stimulating hormone ratio, gonadotropin concentrations in the first morning-voided urine and in 24hour urine, have been proposed to replace the GnRH stimulation test. ${ }^{2)}$ However, basal serum gonadotropin levels have diurnal variation and lower sensitivity and specificity. In addition, although measuring urinary concentrations of gonadotropins is noninvasive, convenient, and easy, there is a limit for testing the first-voided urine in the morning or collecting 24hour urine in an outpatient clinic. This recent study shows that when urinary gonadotropin concentrations are measured, it is sufficient to measure random urine, not first-voided urine or 24-hour collecting urine. Especially because randomly measured urinary concentrations of gonadotropins significantly decreases during GnRH agonist treatment, it might be useful to monitor during GnRH agonist treatment in CPP girls. ${ }^{1,3)}$

However, even though urinary gonadotropin concentrations sharply increase in puberty, ${ }^{4)}$ it periodically shows every other day in fluctuating patterns. ${ }^{5}$ In addition, the number of subjects in this study remains small, raising questions about the statistical significance of the data. Therefore, further large-scale prospective studies are needed to confirm the usefulness and value of the randomly measured urinary concentrations of gonadotropins. Nevertheless, based on the results of the present study, $\left.{ }^{1}\right)$ it is worth replacing serum gonadotropin concentration test to monitor the treatment effect, which will alleviate the pain of many CPP girls undergoing GnRH agonist treatment.

\section{Conflict of interest}

No potential conflict of interest relevant to this article was reported.

\section{References}

1. Lee SY, Kim JM, Kim YM, Lim HH. Single random measurement of urinary gonadotropin concentration for screening and monitoring of girls with central precocious puberty. Ann Pediatr Endocrinol Metab 2021;26:178-184.

2. Ab Rahim SN, Omar J, Tuan Ismail TS. Gonadotropin-releasing hormone stimulation test and diagnostic cutoff in precocious puberty: a mini review. Ann Pediatr Endocrinol Metab 2020;25:152-5. 
3. Lucaccioni L, McNeilly J, Mason A, Giacomozzi C, Kyriakou A, Shaikh MG, et al. The measurement of urinary gonadotropins for assessment and management of pubertal disorder. Hormones (Athens) 2016;15:377-84.

4. Demir A, Dunkel L, Stenman UH, Voutilainen R. Agerelated course of urinary gonadotropins in children. J Clin
Endocrinol Metab 1995;80:1457-60.

5. Maesaka H, Tachibana K, Adachi M, Okada T. Monthly urinary gonadotropin and ovarian hormone excretory patterns in normal girls and female patients with idiopathic precocious puberty. Pediatr Res 1996;40:853-60. 